•研究报告・

\title{
南亚热带常绿阔叶林树木多样性与生物量和生产 力的关联及其影响因素
}

朱杰 ${ }^{1,2,3,4,5}$, 吴安驰 ${ }^{1,2}$, 邹顺 ${ }^{6}$, 熊金金 ${ }^{1}$, 刘世忠 ${ }^{1}$, 褚国伟 ${ }^{1}$, 张倩媚 ${ }^{1}$, 刘菊秀 ${ }^{1}$, 唐旭 利 ${ }^{1}$, 间俊华 ${ }^{1}$, 张德强 $^{1}$, 周国逸 ${ }^{* *}$

1. 中国科学院华南植物园，广州 510650；2. 中国科学院大学资源与环境学院，北京 100049; 3. 广东省国土资源测绘院，广州 510663; 4. 自然资源部华南热带亚热带自然资源重点实验室，广州 510663; 5. 广东省自然资源科技协同创新中心，广州 510663; 6. 贵州工程应用 技术学院, 贵阳 551700; 7. 南京信息工程大学应用气象学院, 南京 210044

摘要: 生物多样性和生态系统功能的关系直接或间接地影响着生产力, 是生态学研究的关键问题。本研究旨在定量探讨亚热 带自然林演替后期森林生态系统树木多样性与生物量或生产力的关系。本研究基于中国南亚热带长期永久性样地的群落调查 数据以及地形和土壤养分数据, 分析了南亚热带常绿阔叶林树木多样性与生物量和生产力的关联及其影响因素。相关性分析 结果表明, 物种多样性与生物量呈显著负相关, 与生产力呈显著正相关; 结构多样性与生物量呈显著正相关, 与生产力呈显 著负相关。此外, 不同环境因子对多样性、生物量和生产力的影响具有显著差异, 其中土壤含水量对生产力有显著影响, 物 种多样性指标与部分地形和土壤因子均有相关性，而群落结构多样性指标与土壤因子的相关性更强。方差分解结果表明，结 构多样性对生物量和生产力的单独效应的解释率最大，分别为35.39\%和5.21\%; 其次是结构多样性和物种多样性的共同效应, 对生物量和生产力的解释率分别为13.66\%和3.53\%; 地形和土壤因子的解释率较小。同时, 结构方程结果也表明, 结构多样性 对生物量有较强的直接正影响; 生物量对生产力有强烈的直接负影响, 结构多样性通过增加生物量明显地减少了生产力; 土 壤和地形因子主要是通过物种和结构多样性间接影响生物量和生产力。综上, 本研究认为在南亚热带森林演替顶极群落中, 群落结构复杂性和物种多样性的提高对促进群落生产力和生物量具有重要作用。

关键词: 生物量; 生产力; 土壤养分; 物种多样性; 结构多样性; 地形

朱杰, 吴安驰, 邹顺, 熊釒金, 刘世忠, 褚国伟, 张倩媚, 刘菊秀, 唐旭利, 问俊华, 张德强, 周国逸 (2021) 南亚热带常绿阔叶林树木多样性与生物量和 生产力的关联及其影响因素. 生物多样性, 29, 1435-1446. doi: 10.17520/biods.2021014.

Zhu J, Wu AC, Zou S, Xiong X, Liu SZ, Chu GW, Zhang QM, Liu JX, Tang XL, Yan JH, Zhang DQ, Zhou GY (2021) Relationships between tree diversity and biomass/productivity and their influence factors in a lower subtropical evergreen broad-leaved forest. Biodiversity Science, 29, 1435-1446. doi: 10.17520/biods.2021014

\section{Relationships between tree diversity and biomass/productivity and their influence factors in a lower subtropical evergreen broad-leaved forest}

Jie Zhu ${ }^{1,2,3,4,5}$, Anchi Wu ${ }^{1,2}$, Shun Zou ${ }^{6}$, Xin Xiong ${ }^{1}$, Shizhong Liu $^{1}$, Guowei Chu ${ }^{1}$, Qianmei Zhang ${ }^{1}$, Juxiu Liu ${ }^{1}$, Xuli Tang $^{1}$, Junhua Yan ${ }^{1}$, Deqiang Zhang ${ }^{1}$, Guoyi Zhou

1 South China Botanical Garden, Chinese Academy of Sciences, Guangzhou 510650

2 College of Resources and Environment, University of Chinese Academy of Sciences Beijing, 100049

3 Surveying and Mapping Institute Lands and Resource Department of Guangdong Province, Guangzhou 510663

4 Key Laboratory of Natural Resources Monitoring in Tropical and Subtropical Area of South China, Ministry of Natural Resources, Guangzhou 510663

5 Guangdong Science and Technology Collaborative Innovation Center for Natural Resources, Guangzhou 510663

6 Guizhou University of Engineering Science, Guiyang, 551700

7 School of Applied Meteorology, Nanjing University of Information Science \& Technology, Nanjing 210044

\section{ABSTRACT}

Aim: The relationship between biodiversity and ecosystem function contribute to productivity, both directly and

收稿日期: 2021-01-12; 接受日期: 2021-10-25

基金项目：国家自然科学基金(42130506; 42071031)、广东省科技计划项目(2018B020207002)和广东省林业科技创新平台项目(2020-KYXM-09)

* 通讯作者 Author for correspondence. E-mail: gyzhou@scib.ac.cn 
indirectly. Therefore, it is a critical issue of ecology. The aim of this study is to quantitatively explore the relationship between tree diversity and biomass or productivity of subtropical natural forest ecosystems in late successional stages.

Methods: Based on long-term community surveys of topographies and soil nutrients from permanent forest ecosystems in the subtropical evergreen broad-leaved forest, we analyzed the relationship between tree diversity and productivity/biomass and their influencing factors. In this analysis, the correlations between tree diversity and each factor were evaluated using a Pearson correlation analysis. The single and shared effects of each factor were quantified by variance partitioning analysis (VPA). In addition, the relationships between soil nutrients and topographies and their effects on productivity and biomass were further evaluated, either directly or indirectly, through species and structural diversity by using a structural equation model (SEM).

Result: Species diversity displayed a negative correlation with biomass and a positive correlation with productivity. Contrarily, structural diversity was positively correlated with biomass and negatively correlated with productivity. The effects of environment factors on tree diversity and biomass/productivity were varied. Specifically, soil moisture had a significant effect on productivity, species diversity was correlated with soil nutrients and topographies, and structural diversity was strongly related to soil nutrients. The variance partitioning analysis results indicated that the single effect of structural diversity explained the largest portion of variance in biomass (35.39\%) and productivity (5.21\%), followed by the shared effect of structural and species diversity on biomass (13.66\%) and productivity (3.53\%). Soil nutrients and topographies explained less variation in productivity and biomass. The structural equation results analysis indicated that structural diversity had a direct positive effect on biomass, and biomass had a strong direct negative effect on productivity. Structural diversity indirectly reduced productivity by increasing biomass. Soil nutrients and topographies were mainly affected by biomass, while productivity indirectly affected tree species and structural diversity.

Conclusions: These results indicate that (1) species diversity had the greatest direct effect on productivity, and structural diversity had the greatest direct effect on biomass in southern subtropical evergreen broad-leaved forests; (2) species diversity explained productivity better than structural diversity, while structural diversity explained biomass accumulation better than species diversity; and (3) both species diversity and structural diversity can be affected by soil nutrients and topographies.

Key words: biomass; productivity; soil nutrients; species diversity; structural diversity; topographies

生物多样性和生产力是生态系统的核心指标, 是实现生态系统服务与功能的根本所在(Tilman et $\mathrm{al}, 2014)$ 。在全球气候变化的大环境下, 生物多样性 锐减, 生态系统保护面临空前困境(Chapin et al, 2000)。因此, 保护生物多样性, 提高和恢复生态系 统功能具有重要意义(Wilsey \& Potvin, 2000)。在生 物多样性和生态系统功能的关系中, 多样性和生产 力之间的关系及其维持机制是全球科学家所关注 的核心问题之一(Loreau et al, 2001; 贺金生等, 2003)。大量研究表明物种多样性与生产力呈正相关 (Wilson et al, 1990; Tilman \& Downing, 1994; Ouyang et al, 2016; 吴初平等, 2018), 但负相关 (Liang et al, 2007)、驼峰关系(Loreau et al, 2001)和不 相关(Grace et al, 2016)也有报道。森林生态系统中 生物量和生产力之间既有联系又有区别, 有研究表 明, 在森林生态系统中生产力高的物种其碳储存能 力通常要低于生产力低的物种(吴初平等, 2018), 且 生物量和生产力对物种丰富度的响应也有差异 (Cardinale et al, 2006)。综上, 关于天然林中物种多 样性对生产力和生物量的影响及其维持机制仍存
在较大争议(Zhang \& Chen, 2015; Fotis et al, 2017)。 为了加深对复杂自然生态系统中多样性与生产力 关系和机制的理解, 亟需进一步加强多样性与生产 力和生物量关系的研究。

同时, 群落结构多样性对森林生产力的影响也 非常重要(Liang et al, 2007; Lei et al, 2009; Long \& Shaw, 2010 )。结构多样性通常被定义为空间分布、 物种多样性和树木尺寸变化(如树木高度和直径)的 一种或多种组合(Lei et al, 2009)。复杂的结构可以 提高树木对光的捕获和利用效率, 并提高树木利用 水和土壤养分的效率, 还可以促进森林生态系统中 生物量的积累(Hardiman et al, 2011; Wang et al, 2011)。结构多样性除了能表征群落的空间分布, 也 可以反映群落总体的生物多样性, 不同物种间的固 有差异以及同种或异种个体间的不对称竞争有助 于树木在空间中形成多元配置, 提高资源利用效率 和生产力(Yachi \& Loreau, 2007; Lei et al, 2009)。研 究表明, 个体之间的差异、立地密度、林龄、最大 径级和大树密度等林分结构属性都会改变森林生 物量和生产力格局的多个方面(Paoli et al, 2008; 
Slik et al, 2013; Zhang \& Chen, 2015; Yuan et al, 2018)。物种多样性和林分结构属性的变化也直接关 系到林分的生长和管理实践(Kant, 2002; Liang et al, 2005; Lei et al, 2009)。综上所述, 群落结构多样性对 生产力和生物量的影响机制亟待进一步阐明。

植物群落的分布格局是不同尺度上气候、土 壤、地形等环境因子综合作用的结果(Ricklefs et al, 1999)。植被的生产力也受到物种组成和地理环境等 因素的影响(Doherty et al, 2011; Zhang et al, 2012)。 土壤肥力和地形的差异都是影响植物群落结构和 生产力的关键因素(Perroni-Ventura et al, 2006; Long et al, 2012)。研究表明高的土壤养分利用率可以促 进植物快速生长, 进而对树木的生产力具有积极效 应(Quesada et al, 2012; Prado-Junior et al, 2016)。相 比于土壤贫瘦的森林, 土壤肥沃的森林中生物多样 性对生产力的影响更大(Paquette \& Messier, 2011)。 此外, 海拔、坡度和坡向等地形特征造成的环境差 异也会影响树种的分布、生物量的积累和生产力的 变化( Valencia et al, 2009; Murphy et al, 2015)。由于 环境条件、时空尺度和群落类型等因素的差异, 物 种多样性和生产力之间的关系尚未得到一致的结 论(Willig, 2011)。因此, 复杂的天然林生态系统中生 物多样性与生产力的关联及其与环境因素的关系 有待进一步阐明(谭凌照等, 2017)。

鼎湖山地处中国南亚热带典型季风气候区, 其 地带性植被常绿阔叶林具有400多年的保护历史(方 运霆等, 2003; 张亚茹等, 2014)。近年来该区域受气 候变化等多重因素的影响出现了氮沉降增加和磷 限制加剧等问题, 亟需加强对该生态系统生物多样 性与生物量和生产力关系的研究(方运霆等, 2005; 黄文娟等, 2009; Lie et al, 2019)。分析南亚热带常绿 阔叶林树木多样性与生产力和生物量的关联及其 影响因素, 对于南亚热带常绿阔叶林生产力的提高 和生物多样性的保护尤为重要。本研究以鼎湖山南 亚热带常绿阔叶林群落为研究对象, 结合多种统计 方法分析和评估树木多样性与生物量和生产力之 间的关联及其影响因素。主要探讨以下问题: (1)生 产力是否受物种多样性和林分结构多样性的影响? (2)生物量是否受物种多样性和林分结构多样性的 影响? 是否与物种多样性和林分结构多样性对生 产力的影响一致? (3)环境因子如何影响物种多样性 和林分结构多样性?

\section{材料与方法}

\section{1 研究地概况}

鼎湖山国家级自然保护区位于广东省肇庆市 鼎湖区 $\left(23.16^{\circ}-23.19^{\circ} \mathrm{N}, 112.51^{\circ}-112.56^{\circ} \mathrm{E}\right)$, 总面 积 1,155 ha, 主要地形为丘陵和低山, 最高峰鸡笼 山海拔1,000.3 m, 属中国南亚热带季风湿润型气候 (熊金等, 2016)。年平均气温 $22.5^{\circ} \mathrm{C}$, 最冷月 $(1$ 月)和 最热月(7月)平均气温分别为 $13.8^{\circ} \mathrm{C}$ 和 $28.8^{\circ} \mathrm{C}$ 。降水 量1,714 mm，4-9月为湿季，10月至翌年3月为旱季， 干湿季分明。年平均蒸发量 $1,115 \mathrm{~mm}$, 年平均相对 湿度 $82 \%$, 灾害性天气为寒潮和台风。土壤母质主 要为泥盆纪砂岩、砂页岩、页岩和石英砂岩, 主要 土壤类型为赤红壤和红壤。该地区主要的植被是南 亚热带常绿阔叶林, 乔木层优势种主要为木荷 (Schima superba)、锥(Castanopsis chinensis)、鼎湖 血桐(Macaranga sampsonii)、香楠(Aidia canthioides) 等。灌木层优势种主要为柏拉木 (Blastus cochinchinensis)、九节(Psychotria rubra)、罗伞树 (Ardisia quinquegona)等。草本层优势种主要是淡竹 叶 (Lophatherum gracile)、芒萁 (Dicranopteris dichotoma)、沙皮萍(Hemigramma decurrins)等。

\section{2 样地设置与群落调查}

本研究选取鼎湖山南亚热带常绿阔叶林为研 究对象, 该森林样地位于低山中坡, 具有 400 多年 的保护历史, 处于演替顶极群落阶段, 郁闭度约 $95 \%$ 。样地面积为 1 ha $(100 \mathrm{~m} \times 100 \mathrm{~m})$, 群落调查时 先将样地划分为 100 个 $10 \mathrm{~m} \times 10 \mathrm{~m}$ 的II级样方, 每 个II级样方再划分为 4 个 $5 \mathrm{~m} \times 5 \mathrm{~m}$ 的III级样方(共 400 个)。对样地内所有胸径(DBH) $\geq 1 \mathrm{~cm}$ 的木本植 物进行调查, 记录其种名、胸径、树高、生长状态 和空间坐标, 每个植株给定一个唯一的编号以便后 续进行追踪测定。2010年和2015年我们在样地内共 调查了 8,084 棵木本植物, 隶属37科 60 属。

\section{3 土壤取样和地形指标测定}

在每个III级样方内0-30 $\mathrm{cm}$ 深的土壤中利用土 钻采集 5 个样品(对角线上), 去掉上层浮土, 混合均 匀。取一部分迅速装于密封袋内, 剩余部分装在土 壤袋中带回实验室测定土壤含水量(WC)、有机碳 $(\mathrm{C}) 、 \mathrm{pH}$ 值 $(\mathrm{pH})$ 、全氮 $(\mathrm{N})$ 、铵态氮 $\left(\mathrm{NH}_{4}^{+}\right)$、懒效磷 $(\mathrm{AP})$ 和速效钾(AK) 7 个指标(刘光菘等, 1996)。用标尺、 重锤和激光笔配合测定各III级样方边缘的相对高 
差, 以整个样地海拔最低的一边为基线, 推算每个 样地的相对海拔。以样地最低点作为零点, 推算出 每个III级样方的顶角在样地中的相对海拔。每个 III 级样方的相对海拔值由四个顶角坐标的平均值表 示。每个样地的地形变量包括相对海拔 $(R A)$ 、东西 坡度 $\left(S_{\mathrm{EW}}\right)$ 、南北坡度 $\left(S_{\mathrm{NS}}\right)$ 、东西相对高差 $\left(R D_{\mathrm{EW}}\right)$ 和南北相对高差 $\left(R D_{\mathrm{NS}}\right)($ 表 1$)$ 。

\section{4 多样性测度}

\subsection{1 物种多样性}

本研究选用物种丰富度 $(R) 、$ Shannon-Wiener多 样性指数 $\left(H^{\prime}\right) 、$ Simpson多样性指数 $(D)$ 和Pielou均匀 度指数 $(E) 4$ 个常用指数测度群落物种多样性。计算 公式如下(Whittaker, 1972; Pielou, 1975):

$$
\begin{aligned}
& R=S \\
& H^{\prime}=-\sum P_{i} \ln P_{i} \\
& D=1-\Sigma P_{i}^{2} \\
& E=H^{\prime} / \ln S
\end{aligned}
$$

式中, $S$ 为样方中物种数量, 选取2015年和2010年同 一样方的平均物种数; $P_{i}$ 为群落中第 $i$ 个物种的重要 值。利用重要值(importance value, $I V$ )来反映每个物 种在群落中的相对重要性, 计算公式为: $I V=$ (相对 多度 + 相对优势度 $) / 2$ 。

\subsection{2 结构多样性}

本研究选用胸径和树高的标准偏差(standard deviation, $S D$ )、变异系数(coefficient of variation, $C V$ ) 和基尼系数(Gini coefficient, GC) 3 个常用指数测度 群落结构多样性。其中, 胸径变异系数是描述林分 分布频率的常用指标，系数值越大表示林木大小分 化程度越高; 基尼系数是描述个体胸径或树高偏离 绝对均匀程度的指标，当个体间没有差异时取值为 0 ，当差异最大化时取值无限接近于1 (Lexerød \& Eid, 2006)。计算公式如下(谭凌照等, 2017; Schnabel et al, 2019):

\begin{tabular}{|c|c|c|c|}
\hline 变量 Variables & 范围 Range & 平均值 Mean & 标准偏差 SD \\
\hline 物种丰富度 Species richness $(R)$ & $6.00-21.50$ & 12.86 & 3.69 \\
\hline Shannon-Wiener多样性指数 Shannon-Wiener diversity index $\left(H^{\prime}\right)$ & $1.23-2.68$ & 2.07 & 0.31 \\
\hline Simpson多样性指数 Simpson diversity index $(D)$ & $0.62-0.91$ & 0.82 & 0.06 \\
\hline Pielou均匀度指数 Pielou evenness index $(E)$ & $0.59-0.98$ & 0.82 & 0.08 \\
\hline 胸高断面积标准偏差 Basal area standard deviation $\left(S D_{b a}\right)$ & $14.83-4,696.75$ & 333.41 & 678.16 \\
\hline 胸高断面积变异系数 Basal area coefficient of variation $\left(C V_{b a}\right)$ & $86.49-2,069.74$ & 486.98 & 371.29 \\
\hline 胸高断面积基尼系数 Basal area Gini coefficient $\left(G C_{b a}\right)$ & $0.55-1.00$ & 0.82 & 0.10 \\
\hline 树高标准偏差 Height standard deviation $\left(S D_{h}\right)$ & $1.49-8.40$ & 4.14 & 1.77 \\
\hline 树高变异系数 Height coefficient of variation $\left(C V_{h}\right)$ & 28.73-147.37 & 79.32 & 30.73 \\
\hline 树高基尼系数 Height Gini coefficient $\left(G C_{h}\right)$ & $0.18-0.54$ & 0.35 & 0.08 \\
\hline 坡度(南北) Slop degree (Northsouth) $\left(S_{\mathrm{NS}}\right)\left(^{\circ}\right)$ & $0.00-89.61$ & 10.52 & 11.86 \\
\hline 坡度(东西) Slop degree(Eastwest) $\left(S_{\mathrm{EW}}\right)\left(^{\circ}\right)$ & $4.72-39.00$ & 26.79 & 5.78 \\
\hline 相对高差(南北) Relative dispersion (Northsouth) $\left(R D_{\mathrm{NS}}\right)(\mathrm{m})$ & $0.00-3.07$ & 0.95 & 0.83 \\
\hline 相对高差(东西) Relative dispersion (Eastwest) $\left(R D_{\mathrm{EW}}\right)(\mathrm{m})$ & $0.39-4.09$ & 2.58 & 0.62 \\
\hline 相对海拔 Relative altitude $(R A)(\mathrm{m})$ & $4.50-66.20$ & 33.42 & 15.47 \\
\hline 土壤含水量 Water content (WC) & $0.20-0.30$ & 0.24 & 0.02 \\
\hline $\mathrm{pH}$ 值 $\mathrm{pH}$ value & $3.74-4.05$ & 3.85 & 0.06 \\
\hline 有机碳 Organic carbon (C) (mg/g) & $18.79-42.75$ & 26.18 & 4.37 \\
\hline 全氮 Total nitrogen (TN) (mg/g) & $1.61-3.62$ & 2.14 & 0.33 \\
\hline 铵态氮 Ammonium nitrogen $\left(\mathrm{NH}_{4}^{+}\right)(\mathrm{mg} / \mathrm{g})$ & $0.00-0.01$ & 0.01 & 0.00 \\
\hline 速效磷 Available phosphorus (AP) (mg/g) & $0.00-0.01$ & 0.00 & 0.00 \\
\hline 速效钾 Available potassium (AK) (mg/g) & $0.08-0.23$ & 0.12 & 0.03 \\
\hline
\end{tabular}

表1 南亚热带常绿阔叶林样地基础变量信息统计表

Table 1 The statistical information of basic variables in the sampling plot in a lower subtropical evergreen broad-leaved forest 


$$
\begin{aligned}
& C V_{b a}=100 \times \frac{S D_{b a}}{\bar{x}_{b a}} ; C V_{h}=100 \times \frac{S D_{h}}{\bar{x}_{h}} \\
& G C_{b a}=\frac{\sum_{j=1}^{n}(2 \times j-n-1) \times b a_{j}}{\sum_{j=1}^{n}(n-1) \times b a_{j}} ; \\
& G C_{h}=\frac{\sum_{j=1}^{n}(2 \times j-n-1) \times h_{j}}{\sum_{j=1}^{n}(n-1) \times h_{j}}
\end{aligned}
$$

式中, $b a_{j}$ 是样方内按大小升序第 $j$ 棵树的胸高断面 积, $h_{j}$ 是样方内按大小升序第 $j$ 棵树的树高 $(\mathrm{m}), \bar{x}_{b a}$ 指样方中所有个体胸高断面积的平均值, $\bar{x}_{h}$ 指样 方中所有树高的平均值, $j$ 是树的等级, 从1 1 升到 $n$ 。

其中胸径和树高选取2015年和2010年同一个体的 平均胸径和平均树高。

\section{5 生物量和生产力的计算}

生物量(温达志等, 1997)和生产力(Lasky et al, 2014; 吴初平等, 2018)的计算如下:

生物量 $(B): B=\mathrm{a} \times \mathrm{DBH}^{\mathrm{b}}$

生产力 $(P): P=\left(\right.$ Biomass $_{2015} /$ Biomass $\left._{2010}\right) / t$

式中, $\mathrm{DBH}$ 为胸径 $(\mathrm{cm})$, $\mathrm{a}$ 和 $\mathrm{b}$ 是回归系数。考虑到地 形和土壤数据是两年份之间的调查数据, 本研究的 生物量采用2015年和2010年的平均生物量。考虑到 样方内起始的生物量可能会反过来影响样方内生 物量的变化值, 本研究用生物量的相对变化量表示 群落的初级生产力 (文中统称为 “生产力”)。为了保 证数据的正态性, 对生物量和生产力的数据进行对 数转换。Biomass 2015 为2015年调查时样方内所有个 体生物量之和, Biomass 2010 为2010年调查时样方内 所有个体生物量之和, $t$ 为从2010年到2015年的时间 间隔。

\section{6 数据统计和分析}

首先, 分别对物种多样性、结构多样性、土壤 养分和地形因子进行主成分分析 (principal component analysis, PCA), 再运用一元回归模型检 验树木多样性与生物量和生产力之间的关系。其次, 利用Pearson相关性分析解释物种多样性、结构多样 性、生产力、生物量、土壤养分和地形因子变化之 间的相关关系, 统计显著性水平设为 $P<0.05$ 。最后, 运用方差分解 (variation partitioning analysis, VPA) 解释每组因子的单独效应和共同效应, 利用韦恩图 表示各因子对生物量和生产力变化的相对贡献。同
时, 利用结构方程模型(structural equation modeling, SEM)检验土壤养分和地形因子通过物种多样性和 结构多样性对生产力和生物量的直接和间接效应。 采用最大似然法对结构方程模型进行拟合, 利用比 较拟合指数 $(C F I)$ 、渐进残差均方和平方根 $(R M S E A) 、$ 显著性概率值 $(P)$ 评价模型优度, 拟合优 度的临界值为 $C F I>0.9, R M S E A<0.08, P>0.05$, $A I C$ 越小越好(温纯和金光泽, 2019)。数据整理与计 算使用Excel 2016, 结构方程模型构建采用AMOS 21.0, 统计分析及作图在R 3.3.3软件中完成。

\section{结果}

\section{1 树木多样性与生产力和生物量之间的关系}

利用主成分分析对物种多样性指标和结构多 样性指标进行聚类分析(表2)。结果表明物种多样性 第一主轴的解释率为 $73.4 \%$, 第二主轴的解释率为 25.5\% (图1a)。结构多样性第一主轴的解释率为 $70.4 \%$, 第二主轴的解释率为 $15.5 \%$ (图1b)。其第一 主轴载荷量均大于 0.7 , 说明第一个主轴能代表物 种多样性和结构多样性的变化。树木多样性与生物 量和生产力的一元线性回归结果显示, 在南亚热带 常绿阔叶林中生产力随着物种多样性的增加而极 显著增加 $\left(R^{2}=0.12, P<0.01\right.$, 图1c), 而生物量随着 物种多样性的增加极显著减少 $\left(R^{2}=0.10, P<0.01\right.$, 图1c)。相反, 生产力随着结构多样性的增加而减少 $\left(R^{2}=0.20, P<0.001\right.$, 图 $\left.1 \mathrm{~d}\right)$, 生物量随着结构多样 性的增加而极显著增加 $\left(R^{2}=0.52, P<0.01\right.$, 图1d)。

\section{2 环境因子与树木多样性、生产力和生物量的相} 关性

Person相关性分析结果显示, 生产力与土壤含 水量显著负相关 $(P<0.05)$, 而生物量与地形和土壤 养分均没有显著的相关关系(图2)。物种丰富度与有 机碳、速效磷和速效钾呈显著负相关 $(P<0.05)$, 与 坡度(南北)和相对高差(南北)极显著负相关 $(P<$ $0.01)$, 与全氮呈负相关 $(P<0.001)$ 。Shannon-Wiener 多样性指数与坡度 (南北) 显著负相关 $(P<0.05)$, 与 全氮极显著负相关 $(P<0.01)$, 与铵态氮极显著正相 关 $(P<0.01)$ 。Simpson多样性指数与相对高差(南北) 显著负相关 $(P<0.05)$, 与铵态氮显著正相关, 与坡 度(南北)和全氮极显著负相关 $(P<0.01)$ 。Pielou均匀 度指数与土壤含水量和铵态氮显著正相关 $(P<$ 0.05)。胸高断面积变异系数与土壤含水量和铵态氮 
显著负相关 $(P<0.05)$ 。胸高断面积基尼系数与速效 磷呈显著负相关 $(P<0.05)$ 。树高标准偏差与相对海 拔、土壤含水量和速效磷呈显著负相关 $(P<0.05)$; 树高变异系数与有机碳、铵态氮、速效磷和速效钾 呈显著负相关 $(P<0.05)$, 与土壤含水量极显著负相 关 $(P<0.01)$ 。树高基尼系数与相对海拔、土壤含水 量、有机碳和速效磷呈显著负相关 $(P<0.05)$ (图2)。 2.3 环境因子对生产力和生物量的直接和间接影响 方差分解分析结果表明，结构多样性对生产力 的单独效应的解释率最大, 为 $5.21 \%$; 结构多样性 和物种多样性共同效应的解释率次之，为 $3.53 \%$; 地形与结构多样性共同效应和土壤养分与结构多 样性共同效应的解释率较小, 分别为 $1.09 \%$ 和 $0.19 \%$ (图3a)。结构多样性对生物量单独效应的解释率最 大，为35.39\%; 结构多样性和物种多样性共同效应 的解释率次之，为 $13.66 \%$; 地形与结构多样性共同 效应和土壤养分与结构多样性共同效应的解释率 较小, 分别为3.52\%和3.49\% (图3b)。进一步利用结 构方程模型进行分析，结果表明各变量分别解释生 产力和生物量的变化的34\%和52\% (图3c)。物种多 样性对生产力有显著的直接影响 $(P<0.05)$, 反映作 用大小的路径系数为 0.18 。结构多样性对生物量具 有直接影响 $(P<0.001$, 路径系数为 0.72$)$ 。各变量分 别解释了物种多样性和结构多样性变化的 $29 \%$ 和 $5 \%$ 。地形和土壤养分对物种多样性具有显著的直接 影响，路径系数分别为 -0.23 和 -0.26 ; 土壤养分对
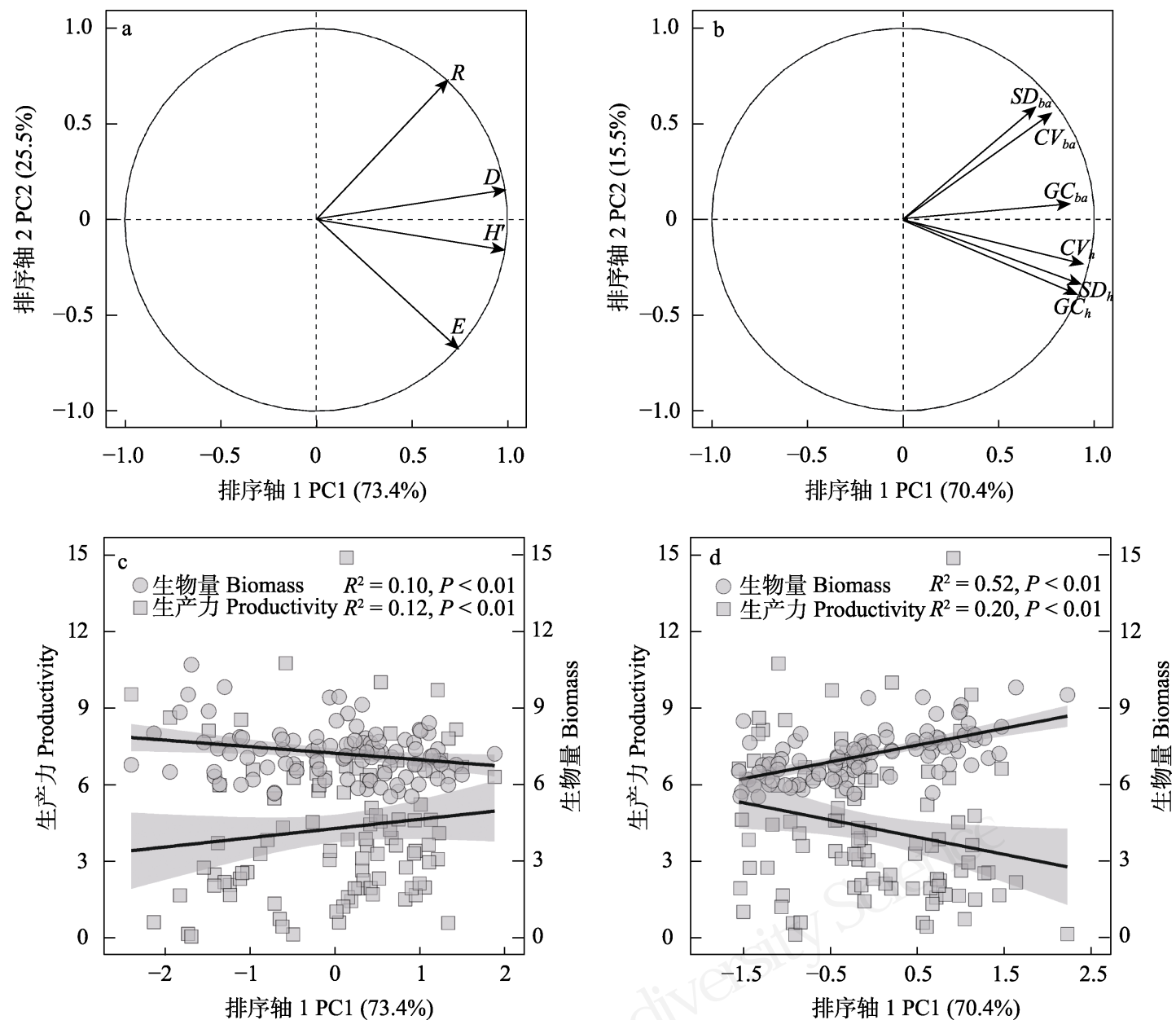

图1 物种多样性和结构多样性与生物量和生产力之间的相关关系。(a) 4个物种多样性指标的第一轴和第二轴载荷量; (b) 6 个结构多样性指标的第一轴和第二轴载荷量; (c)物种多样性第一主轴载荷量与生物量和生产力之间的关系; (d)结构多样性第 一主轴载荷量与生物量和生产力之间的关系。缩写代表含义同表1。

Fig. 1 Relationship between species diversity and structural diversity with plant biomass and productivity. (a) Loadings on the first and second axes of four species diversity indexes; (b) Loadings on the first and second axes of six structural diversity indexes; (c) Relationship between loadings on the first axe of species diversity and plant biomass and productivity; (d) Relationship between loadings on the first axe of structural diversity and plant biomass and productivity. Abbreviations have the same as in Table 1. 
表2 物种多样性、结构多样性、地形和土壤养分主成分的 特征值和贡献率

Table 2 Eigenvalues and contribution rate of species diversity factors, structural diversity factors, topography factors and soil nutrients factors.

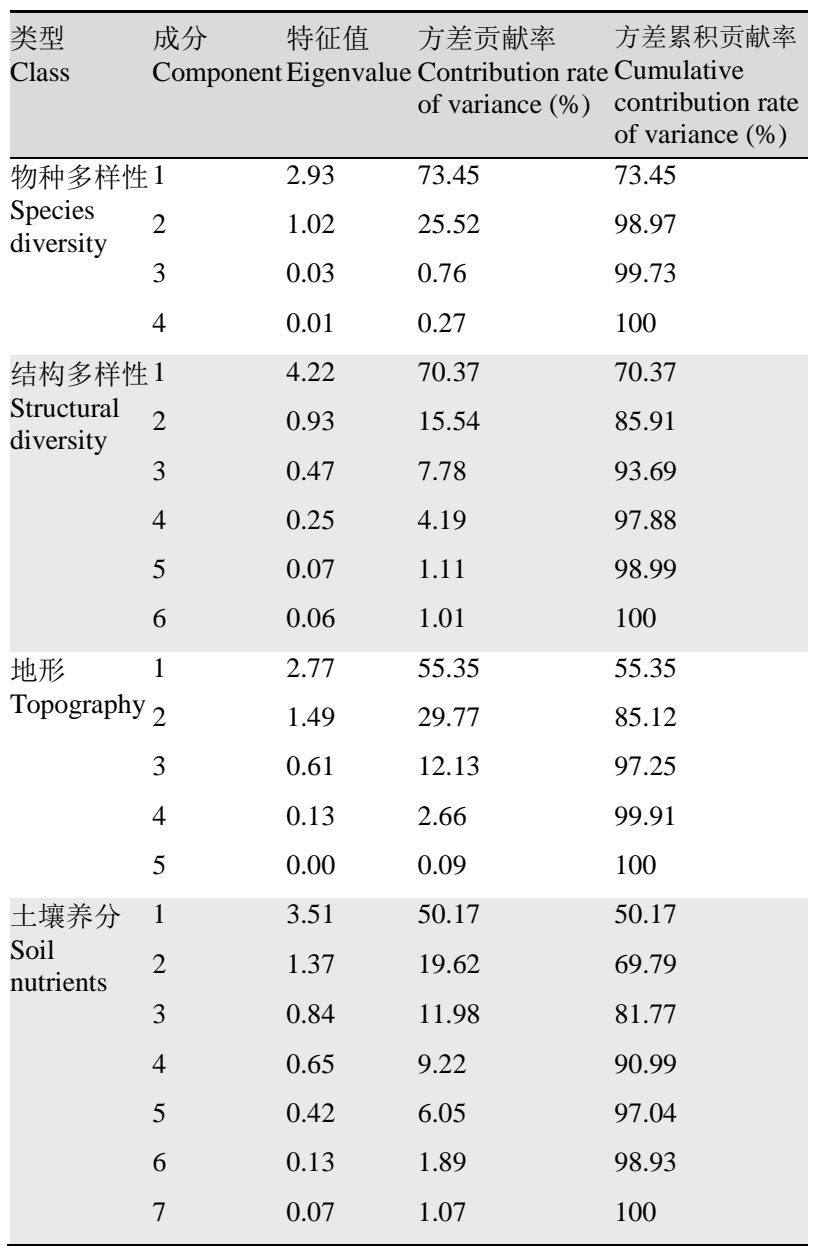

结构多样性具有显著的直接影响, 路径系数为 -0.22 。结构多样性对物种多样性具有极显著影响, 路径系数为 -0.48 ; 生物量对生产力具有极显著影 响, 路径系数为 -0.50 (图3c)。

\section{3 讨论}

\section{1 物种多样性与生产力和生物量的关系}

本研究结果表明物种多样性与生产力呈显著 正相关关系，这与早期生产力与物种多样性关系的 研究结果相似(黄小荣等, 2018; Liu et al, 2019)。这 可能由于鼎湖山南亚热带常绿阔叶林群落郁闭度 较高，林龄超过200年，生态位分化合理，使得空间 资源能够被充分利用，进而增加了植被的生产力 (Loreau et al, 2001; Chen et al, 2016)。并且以往研究

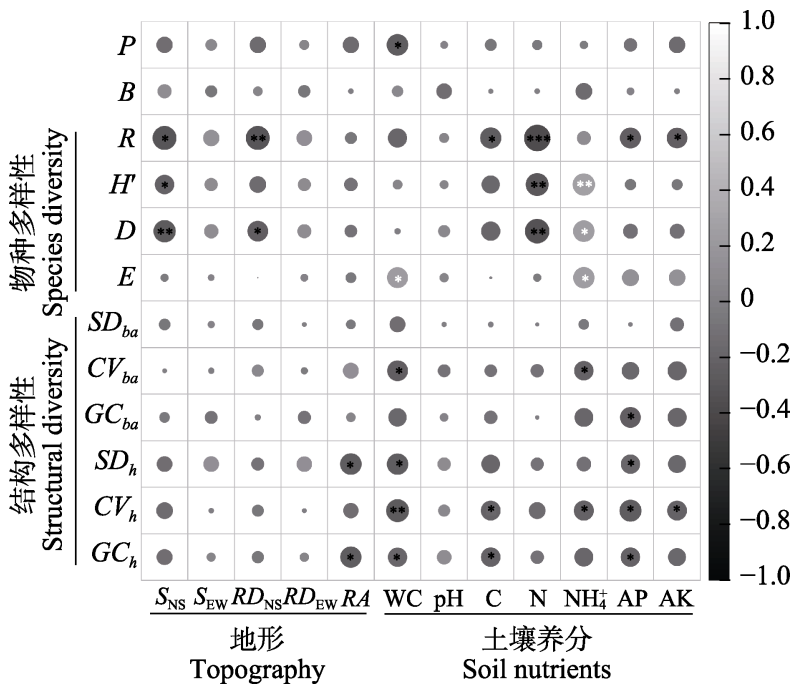

图2 南亚热带常绿阔叶林树木多样性、生物量和生产力与 土壤养分和地形因子之间的 Pearson相关关系。P: Productivity; B: Biomass; 其余缩写代表含义同表1, *, P< $0.05, * *, P<0.01, * * *, P<0.001$, 白色*为正相关, 黑色*为 负相关。

Fig. 2 Pearson correlation between tree species diversity, plant biomass, plant productivity and soil nutrients, topographic factors in subtropical evergreen brebedved forest The letter $\mathrm{B}$ and $\mathrm{P}$ mean biomass and productivity, and other abbreviations have the same as in Table $1, *, P<0.05$, **, $P<$ 0.01 , ***, $P<0.001$, white sign $\left(^{*}\right)$ is positive correlation, black sign $\left(^{*}\right)$ is negative correlation.

表明物种丰富度高的群落更偏向于增加高产物种 进入群落的机会, 而本研究鼎湖山南亚热带常绿阔 叶林已经处于群落演替后期, 高产物种已经形成了 稳定的种间关系，因此高物种多样性也是植被生产 力增加的原因之一(Vile et al, 2006; Cadotte, 2017)。 此外, 鼎湖山南亚热带常绿阔叶林中群落物种多样 性与生物量呈负相关, 该结果与在浙江定海次生林 生物量与物种多样性的结果并不一致(吴初平等, 2018)。这可能由于本研究群落处于近演替顶极阶段 (邹顺等, 2018), 在演替后期南亚热带常绿阔叶林群 落结构较为稳定, 群落的总生物量主要取决于群落 中的高碳储量的物种，高碳储量的物种竞争性导致 更多的生物量积累(Potter \& Woodall 2014; Ouyang et al, 2016)。而鼎湖山常绿阔叶林以往研究显示，过 去30年间群落中大径级个体和不抗旱植物个体数 减少，小径级个体和抗旱或速生植物个体数增加， 使得群落中总生物量呈现下降的趋势, 这可以用来 解释本研究中物种多样性与生物量的负相关关系 (邹顺等, 2018)。相关研究也表明当生物量超过一定 


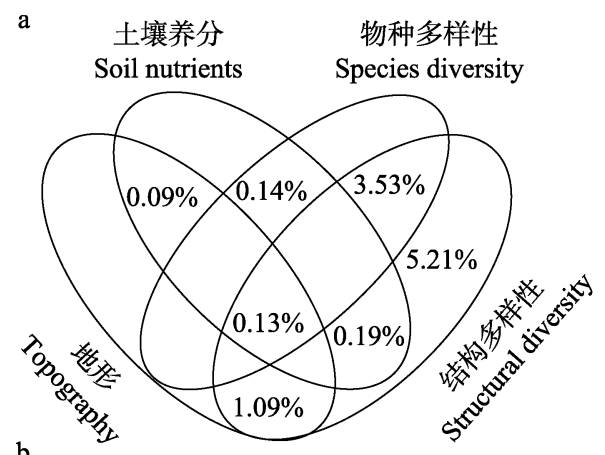

b

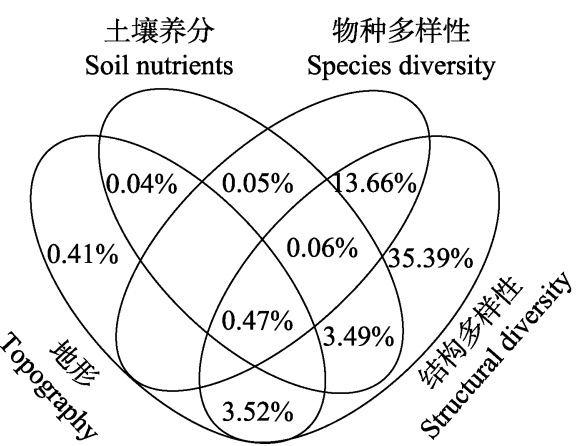

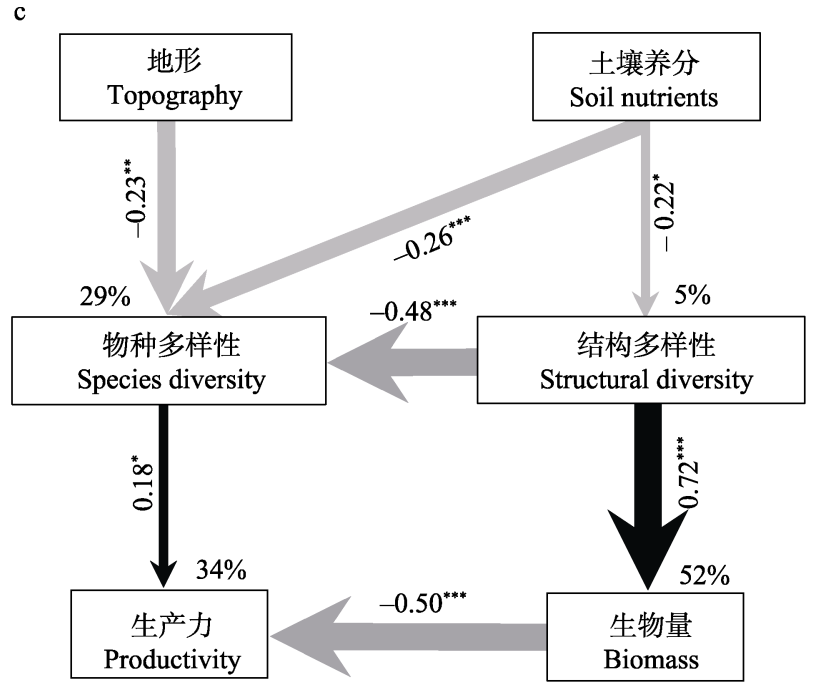

路径系数 Path coefficients

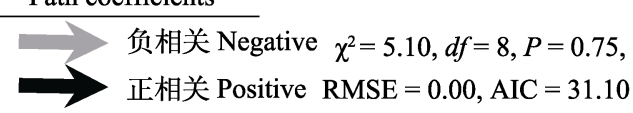

图3 物种多样性、结构多样性、地形和土壤养分对生产力和生物量的影响。(a)方差分解解释各因子对生产力的 单独效应和共同效应; (b)方差分解解释各因子对生物量的单独效应和共同效应; (c)结构方程模型(SEM)解释地 形和土壤养分通过物种多样性和结构多样性对生产力的直接和间接影响 $(N=100)$ 。SEM考虑了所有可能的路径, $\boldsymbol{R}^{2}$ 表示所解释的方差比例。箭头上的数字表示标准化的路径系数。箭头宽度表示路径系数的强度。*, $\boldsymbol{P}<\mathbf{0 . 0 5}, * *$, $\mathbf{P}<0.01$, ***, $\boldsymbol{P}<0.001$ 。

Fig. 3 The impact of species diversity, structural diversity, topography and soil nutrients on plant productivity and biomass. (a) Variation partitioning analysis explains the pure and shared effect of factors on plant productivity. (b) Variation partitioning analysis explains the pure and shared effect of factors on plant biomass. (c) Structural equation model (SEM) reveals the direct and indirect effects of species diversity, structural diversity, topography and soil nutrients on plant productivity and biomass $(N=100)$. The SEM considered all plausible pathways, to increase the degrees of freedom, $R^{2}$ indicates the proportion of variance explained. The numbers on the arrows indicate standardized path coefficients. The arrow width is proportional to the strength of the path coefficients. $*, P<0.05, * *, P<0.01, * * *$, $P<0.001$.

水平时, 物种丰富度与生物量呈显著的负相关(郭 志华等, 2002)。此外, 本研究显示鼎湖山南亚热带 常绿阔叶林生物量与生产力具有负相关关系, 这与 早期在该地区的研究结果一致(Prado-Junior et al, 2016)。这可能是由于环境因素对生物量和生产力关 系的影响(Ma et al, 2010), 本研究群落处于演替后 期阶段, 林分内养分循环处于稳定甚至关闭的状态, 小径级个体虽有生长, 但大径级个体生物量在群落 中占主导地位, 而研究区域内大径级个体受到环境 因素的影响个体数减少, 导致群落生物量与生产力 之间表现出负相关关系。

\section{2 结构多样性与生产力和生物量的关系}

早期研究表明, 群落结构变异在一定程度上反 映了物种在资源利用上的生态位互补, 是促进生产
力的一个重要机制(谭凌照等, 2017)。而鼎湖山南亚 热带常绿阔叶林结构多样性与生物量呈显著的正 相关关系, 与生产力却呈显著的负相关关系。本研 究中结构多样性与生产力的关系与大多数结果不 一致(即结构多样性促进生产力) (谭凌照等, 2017; Lei et al, 2009)。这可能是由研究样地所处的演替阶 段、环境条件和群落结构差异所导致的。南亚热带 常绿阔叶林受到气候条件变化和自然干扰的影响, 群落整体、群落垂直层次内和垂直层次之间的正相 关关系逐渐减弱, 物种间的互补性不足, 对资源的 利用效率下降, 导致群落结构多样性与生产力呈现 负相关关系。这可能是由于鼎湖山常绿阔叶林群落 更新率和死亡率上升, 大径级个体占据主要地位, 虽有小径级个体快速更新提升群落生产力, 但仍然 
不能够弥补大径级个体死亡对群落所造成的影响 (邹顺等, 2018)。群落结构多样性与生物量的正向关 系可以在一定程度上表明结构多样性是促进个体 生物量积累的。一方面群落结构与资源的充分利用 具有密切关系, 竞争和资源的充分利用决定了物种 分布格局的多样化; 另一方面群落结构与物种组成 有密切的关系, 高碳储量的物种占据着群落主要地 位(Potter \& Woodall 2014; Ouyang et al, 2016)。因此, 南亚热带常绿阔叶林群落结构多样性是促进群落 生物量积累的。此外, 本研究显示结构多样性与生 产力和生物量的关系与物种多样性与生产力和生 物量的关系呈相反的趋势, 这可能是由于本研究中 结构多样性对物种多样性具有反向作用, 群落属于 近演替顶极, 群落结构较为稳定, 而近年来该群落 受环境影响个体不断死亡造成群落巨大波动, 影响 了物种组成, 使结构多样性与物种多样性呈现出负 相关关系(邹顺等, 2018)。

\section{3 环境因子对多样性的影响}

地形和土壤养分的变化决定了物种分布、森林 碳库组成和功能的多个方面。自然群落中的物种组 成是在漫长的演化过程中, 物种与环境之间相互依 赖、相互作用, 从而适应当地环境条件所形成的特 定群落结构, 是对环境响应的综合反映(Ricklefs et al, 1999)。我们的研究结果表明地形对植被生产力 和生物量无显著的直接影响, 但对物种多样性具有 显著的负影响, 这与吴初平等(2018)在浙江定海次 生林地的研究具有一定的差异。当然, 群落结构特 性也受区域特征和群落发展阶段的影响(方精云等, 2004; Jafari et al, 2004)。本研究中地形对物种多样 性的影响主要是由于样地的斑块状特征, 坡度呈西 南-东北走向, 从南到北坡度逐渐增加, 而物种多 样性逐渐下降, 导致地形与物种多样性呈现显著的 负相关关系。

土壤和植被的养分状况常作为衡量环境对植 被生长养分限制状况的指标。在热带和亚热带地区, 由于土壤高度风化以及土壤中磷的有效性低等特 点, 土壤养分常常成为该地区限制植物生长的主要 因素(Vitousek \& Howarth, 1991)。本研究中南亚热 带常绿润叶林高土壤有机碳和土壤有效磷含量的 环境条件下, 群落结构复杂; 而高土壤氮含量环境 条件下, 物种多样性较低, 这与早期研究具有相似
的结果(冯健等, 2021)。这可能是由于南亚热带常绿 阔叶林物种多样性和结构多样性主要受土壤有效 磷和氮沉降的影响, 植物生长所需要的土壤有效磷 主要来源于土壤, 植被中土壤有效磷含量越多, 结 构多样性越复杂。并且南亚热带常绿阔叶林由于氮 沉降导致物种多样性逐渐降低(鲁显楷等, 2008)。因 此, 南亚热带常绿榈叶林土壤养分对物种多样性和 结构多样性有明显的影响。

综上, 本研究以未经人为干扰的中国南亚热带 地区的地带性顶极群落为研究对象, 基于森林生态 系统乔木层群落调查数据, 结合样地的地形和土壤 养分, 研究了南亚热带常绿阔叶林树木多样性和生 产力之间的关联及其影响因素。主要结论如下: (1) 物种多样性对生产力有最大直接效应, 结构多样性 对生物量有最大直接效应; (2)在南亚热带常绿润叶 林中物种多样性在解释生产力方面要优于结构多 样性, 而结构多样性在解释生物量积累方面要优于 物种多样性; (3)物种多样性和结构多样性均受地形 和土壤因子的影响。因此, 在南亚热带常绿阔叶林 的经营和管理过程中充分考虑物种多样性和结构 多样性对生产力和生物量的影响, 将有助于更好的 保持南亚热带常绿阔叶林的结构多样性, 以维持演 替顶级群落的生物多样性和快速生长。

\section{参考文献}

Cadotte MW (2017) Functional traits explain ecosystem function through opposing mechanisms. Ecology Letters, 20, 989-996.

Cardinale BJ, Srivastava DS, Duffy EJ, Wright JP, Downing AL, Sankaran M, Jouseau C (2006) Effects of biodiversity on the functioning of trophic groups and ecosystems. Nature, 443, 989-992.

Chapin FS, Zavaleta ES, Eviner VT, Naylor RL, Vitousek PM, Reynolds HL, Hooper DU, Lavorel S, Sala OE, Hobbie SE, Mack MC, Díaz S (2000) Consequences of changing biodiversity. Nature, 405, 234-242.

Chen YX, Wright SJ, Muller-Landau HC, Hubbell SP, Wang YF, Yu SX (2016) Positive effects of neighborhood complementarity on tree growth in a Neotropical forest. Ecology, 97, 776-785.

Doherty JM, Callaway J, Zedler JB (2011) Diversity-function relationships changed in a long-term restoration experiment. Ecological Applications, 21, 2143-2155.

Fang JY, Shen ZH, Cui HT (2004) Ecological characteristics of mountains and research issues of mountain ecology. Biodiversity Science, 12, 1019. (in Chinese with English 
abstract) [方精云, 沈泽昊, 崔海亭 (2004) 试论山地的生 态特征及山地生态学的研究内容. 生物多样性, 12, 10-19.]

Fang YT, Mo JM, Peng SL, Li DJ (2003) Role of forest succession on carbon sequestration of forest ecosystems in lower subtropical China. Acta Ecologica Sinica, 23, 1685-1694. (in Chinese with English abstract) [方运霆, 莫 江明, 彭少麟, 李德军 (2003) 森林演替在南亚热带森林 生态系统碳吸存中的作用. 生态学报, 23, 1685-1694.]

Fang YT, Mo JM, Zhou GY, Xue JH (2005) Response of diameter at breast height increment to $\mathrm{N}$ additions in forests of Dinghushan Biosphere Reserve. Journal of Tropical and Subtropical Botany, 13, 198-204. (in Chinese with English abstract) [方运霆, 莫江明, 周国逸, 薛璟花 (2005) 鼎湖 山主要森林类型植物胸径生长对氮沉降增加的初期响应. 热带亚热带植物学报, 13, 198-204.]

Feng J, Wang QC, Lu AJ, Yu SH, Zheng Y, Wang YC, Chen DS (2021) Study on vegetation diversity and soil characteristics of Larix olgensis uneven-aged mixed stand in eastern Liaoning mountain region. Journal of Northwest A\&F University (Natural Science Edition), 49, 5766. (in Chinese with English abstract) [冯健, 王骞春, 陆爱君, 于 世河, 郑颖, 王月婵, 陈东升 (2021) 辽东山区长白落叶 松异龄混交林植被多样性和土壤特征研究. 西北农林科 技大学学报(自然科学版), 49, 57-66.]

Fotis AT, Murphy SJ, Ricart RD, Krishnadas M, Whitacre J, Wenzel JW, Queenborough SA, Comita LS (2017) Above-ground biomass is driven by mass - ratio effects and stand structural attributes in a temperate deciduous forest. Journal of Ecology, 106, 561-570.

Grace JB, Anderson TM, Seabloom EW, Borer ET, Adler PB, Harpole WS, Hautier Y, Hillebrand H, Lind EM, Pärtel M, Bakker JD, Buckley YM, Crawley MJ, Damschen EI, Davies KF, Fay PA, Firn J, Gruner DS, Hector A, Knops JMH, MacDougall AS, Melbourne BA, Morgan JW, Orrock JL, Prober SM, Smith MD (2016) Integrative modelling reveals mechanisms linking productivity and plant species richness. Nature, 529, 390-393.

Guo ZH, Zang RG, Jiang YX (2002) The formation and maintenance mechanisms of biodiversity and the research techniques for biodiversity. Scientia Sylvae Sinicae, 38, 116-124. (in Chinese with English abstract) [郭志华, 臓润 国, 蒋有绪 (2002) 生物多样性的形成、维持机制及其宏 观研究方法. 林业科学, 38, 116-124.]

Hardiman BS, Bohrer G, Gough CM, Vogel CS, Curtisi PS (2011) The role of canopy structural complexity in wood net primary production of a maturing northern deciduous forest. Ecology, 92, 1818-1827.

He JS, Fang JY, Ma KP, Huang JH (2003) Biodiversity and ecosystem productivity: Why is there a discrepancy in the relationship between experimental and natural ecosystems? Acta Phytoecologica Sinica, 27, 835843. (in Chi nese with English abstract) [贺金生, 方精云, 马克平, 黄建辉
(2003) 生物多样性与生态系统生产力: 为什么野外观测 和受控实验结果不一致? 植物生态学报, 27, 835-843.]

Huang WJ, Liu JX, Tang XL, Huang YH, Liu SZ, Chu GW, Zhou GY (2009) Inorganic nitrogen and available phosphorus concentrations in the soils of five forests at Dinghushan, China. Chinese Journal of Applied and Environmental Biology, 15, 441-447. (in Chinese with English abstract) [黄文娟, 刘菊秀, 唐旭利, 黄钰辉, 刘世 忠, 褚国伟, 周国逸 (2009) 鼎湖山5种森林土壤的无机 氮和有效磷含量. 应用与环境生物学报, 15, 441-447.]

Huang XR (2018) Relationship between plant functional diversity and productivity of Pinus massoniana plantations in Guangxi. Biodiversity Science, 26, 690700. (in Chinese with English abstract) [黄小荣 (2018) 广西马尾松林植物 功能多样性与生产力的关系. 生物多样性, 26, 690-700.]

Jafari M, Chahouki MAZ, Tavili A, Azarnivand H, Amiri GZ (2004) Effective environmental factors in the distribution of vegetation types in Poshtkouh rangelands of Yazd Province (Iran). Journal of Arid Environments, 56, 627-641.

Kant S (2002) The marginal cost of structural diversity of mixed uneven-aged hard maple forests. Canadian Journal of Forest Research, 32, 616-628.

Lasky JR, Uriarte M, Boukili VK, Erickson DL, John Kress W, Chazdon RL (2014) The relationship between tree biodiversity and biomass dynamics changes with tropical forest succession. Ecology Letters, 17, 1158-1167

Lei XD, Wang WF, Peng CH (2009) Relationships between stand growth and structural diversity in spruce-dominated forests in New Brunswick, Canada. Canadian Journal of Forest Research, 39, 1835-1847.

Lexerød NL, Eid T (2006) An evaluation of different diameter diversity indices based on criteria related to forest management planning. Forest Ecology and Management, 222, 17-28.

Liang JJ, Buongiorno J, Monserud RA (2005) Growth and yield of all-aged Douglas-fir-western hemlock forest stands: A matrix model with stand diversity effects. Canadian Journal of Forest Research, 35, 2368-2381.

Liang JJ, Buongiorno J, Monserud RA, Kruger EL, Zhou M (2007) Effects of diversity of tree species and size on forest basal area growth, recruitment, and mortality. Forest Ecology and Management, 243, 116-127.

Lie ZY, Lin W, Huang WJ, Fang X, Huang CM, Wu T, Chu GW, Liu SZ, Meng Z, Zhou GY, Liu JX (2019) Warming changes soil $\mathrm{N}$ and $\mathrm{P}$ supplies in model tropical forests. Biology and Fertility of Soils, 55, 751-763.

Liu B, Chen HYH, Yang J (2019) Linking understory species diversity, community-level traits and productivity in a Chinese boreal forest. Journal of Vegetation Science, 30, 247-256.

Liu GS, Jiang NH, Zhang LD (1996) Soil Physical and Chemical Analysis \& Description of Soil Profiles. Standards Press of China, Beijing. (in Chinese) [刘光菘, 蒋能慧, 张 
连第 (1996) 土壤理化分析与剖面描述. 中国标准出版 社, 北京.]

Long JN, Shaw JD (2010) The influence of compositional and structural diversity on forest productivity. Forestry, 83, $121-128$.

Long WX, Yang XB, Li DH (2012) Patterns of species diversity and soil nutrients along a chronosequence of vegetation recovery in Hainan Island, South China. Ecological Research, 27, 561-568.

Loreau M, Naeem S, Inchausti P, Bengtsson J, Grime JP, Hector A, Hooper DU, Huston MA, Raffaelli D, Schmid B, Tilman D, Wardle DA (2001) Biodiversity and ecosystem functioning: Current knowledge and future challenges. Science, 294, 804-808.

Lu XK, Mo JM, Dong SF (2008) Effects of nitrogen deposition on forest biodiversity: A review. Acta Ecologica Sinica, 28, 5532-5548. (in Chinese with English abstract) [鲁显楷, 莫 江明, 董少峰 (2008) 氮沉降对森林生物多样性的影响. 生态学报, 28, 5532-5548.]

Ma WH, He JS, Yang YH, Wang XP, Liang CZ, Anwar M, Zeng H, Fang JY, Schmid B (2010) Environmental factors covary with plant diversity-productivity relationships among Chinese grassland sites. Global Ecology and Biogeography, 19, 233-243.

Murphy SJ, Audino LD, Whitacre J, Eck JL, Wenzel JW, Queenborough SA, Comita LS (2015) Species associations structured by environment and land-use history promote beta-diversity in a temperate forest. Ecology, 96, 705-715.

Ouyang S, Xiang WH, Wang XP, Zeng YL, Lei PF, Deng XW, Peng CH (2016) Significant effects of biodiversity on forest biomass during the succession of subtropical forest in South China. Forest Ecology and Management, 372, 291-302.

Paoli GD, Curran LM, Slik JWF (2008) Soil nutrients affect spatial patterns of aboveground biomass and emergent tree density in southwestern Borneo. Oecologia, 155, 287-299.

Paquette A, Messier C (2011) The effect of biodiversity on tree productivity: From temperate to boreal forests. Global Ecology and Biogeography, 20, 170-180.

Perroni-Ventura Y, Montaña C, García-Oliva F (2006) Relationship between soil nutrient availability and plant species richness in a tropical semi-arid environment. Journal of Vegetation Science, 17, 719-728.

Pielou EC (1975) Ecological diversity. John Wiley and Sons, New York.

Potter KM, Woodall CW (2014) Does biodiversity make a difference? Relationships between species richness, evolutionary diversity, and aboveground live tree biomass across U.S. forests. Forest Ecology and Management, 321, 117-129.

Prado-Junior JA, Schiavini I, Vale VS, Arantes CS, van der Sande MT, Lohbeck M, Poorter L (2016) Conservative species drive biomass productivity in tropical dry forests. Journal of Ecology, 104, 817-827.
Quesada CA, Phillips OL, Schwarz M, Czimczik CI, Baker TR, Patiño S, Fyllas NM, Hodnett MG, Herrera R, Almeida S, Alvarez Dávila E, Arneth A, Arroyo L, Chao KJ, Dezzeo N, Erwin T, di Fiore A, Higuchi N, Honorio Coronado E, Jimenez EM, Killeen T, Lezama AT, Lloyd G, López-González G, Luizão FJ, Malhi Y, Monteagudo A, Neill DA, Núñez Vargas P, Paiva R, Peacock J, Peñuela MC, Peña Cruz A, Pitman N, Priante Filho N, Prieto A, Ramirez H, Rudas A, Salomão R, Santos AJB, Schmerler J, Silva N, Silveira M, Vásquez R, Vieira I, Terborgh J, Lloyd J (2012) Basin-wide variations in Amazon forest structure and function are mediated by both soils and climate. Biogeosciences, 9, 2203-2246.

Ricklefs RE, Latham R, Qian H (1999) Global patterns of tree species richness in moist forests: Distinguishing ecological influences and historical contingency. Oikos, 86, 369-373.

Schnabel F, Schwarz JA, Dănescu A, Fichtner A, Nock CA, Bauhus J, Potvin C (2019) Drivers of productivity and its temporal stability in a tropical tree diversity experiment. Global Change Biology, 25, 4257-4272.

Slik JWF, Paoli G, McGuire K, Amaral I, Barroso J, Bastian M, Blanc L, Bongers F, Boundja P, Clark C, Collins M, Dauby G, Ding Y, Doucet JL, Eler E, Ferreira L, Forshed O, Fredriksson G, Gillet JF, Harris D, Leal M, Laumonier Y, Malhi Y, Mansor A, Martin E, Miyamoto K, Araujo-Murakami A, Nagamasu H, Nilus R, Nurtjahya E, Oliveira Á, Onrizal O, Parada-Gutierrez A, Permana A, Poorter L, Poulsen J, Ramirez-Angulo H, Reitsma J, Rovero F, Rozak A, Sheil D, Silva-Espejo J, Silveira M, Spironelo W, Steege H, Stevart T, Navarro-Aguilar GE, Sunderland T, Suzuki E, Tang JW, Theilade I, Heijden G, van Valkenburg J, van Do T, Vilanova E, Vos V, Wich S, Wöll H, Yoneda T, Zang RG, Zhang MG, Zweifel N (2013) Large trees drive forest aboveground biomass variation in moist lowland forests across the tropics. Global Ecology and Biogeography, 22, 1261-1271.

Tan LZ, Fan CY, Fan XH (2017) Relationships between species diversity or community structure and productivity of woody-plants in a broad-leaved Korean pine forest in Jiaohe, Jilin, China. Chinese Journal of Plant Ecology, 41, 1149-1156. (in Chinese with English abstract) [谭凌照, 范 春雨, 范秀华 (2017) 吉林蛟河阔叶红松林木本植物物 种多样性及群落结构与生产力的关系. 植物生态学报, 41, 1149-1156.]

Tilman D, Downing JA (1994) Biodiversity and stability in grasslands. Nature, 367, 363-365.

Tilman D, Isbell F, Cowles JM (2014) Biodiversity and ecosystem functioning. Annual Review of Ecology, Evolution, and Systematics, 45, 471-493.

Valencia R, Condit R, Muller-Landau HC, Hernandez C, Navarrete H (2009) Dissecting biomass dynamics in a large Amazonian forest plot. Journal of Tropical Ecology, 25, 473-482. 
Vile D, Shipley B, Garnier E (2006) Ecosystem productivity can be predicted from potential relative growth rate and species abundance. Ecology Letters, 9, 1061-1067.

Vitousek PM, Howarth RW (1991) Nitrogen limitation on land and in the sea: How can it occur? Biogeochemistry, 13, 87-115.

Wang WF, Lei XD, Ma ZH, Kneeshaw DD, Peng CH (2011) Positive relationship between aboveground carbon stocks and structural diversity in spruce-dominated forest stands in New Brunswick, Canada. Forest Science, 57, 506-515.

Wen C, Jin GZ (2019) Effects of functional diversity on productivity in a typical mixed broadleaved-Korean pine forest. Chinese Journal of Plant Ecology, 43, 94-106. (in Chinese with English abstract) [温纯, 金光泽 (2019) 功能 多样性对典型阔叶红松林生产力的影响. 植物生态学报, 43, 94-106.]

Wen DZ, Wei P, Kong GH, Zhang QM, Huang ZL (1997) Biomass study of the community of Castanopsis chinensis + Cryptocarya concinna + Schina superba in a southern China reserve. Acta Ecologica Sinica, 17, 4754. (in Chinese with English abstract) [温达志, 魏平, 孔国辉, 张倩媚, 黄忠良 (1997) 鼎湖山雉栗 + 黄果厚壳桂 + 荷木群落生物量及 其特征. 生态学报, 17, 47-54.]

Whittaker RH (1972) Evolution and measurement of species diversity. Taxon, 21, 213-251.

Willig MR (2011) Biodiversity and productivity. Science, 333, 1709-1710.

Wilsey BJ, Potvin C (2000) Biodiversity and ecosystem functioning: Importance of species evenness in an old field. Ecology, 81, 887-892.

Wilson JB, Lee WG, Mark AF (1990) Species diversity in relation to ultramafic substrate and to altitude in southwestern New Zealand. Vegetatio, 86, 15-20.

Wu CP, Han WJ, Jiang B, Liu BW, Yuan WG, Shen AH, Huang YJ, Zhu JR (2018) Relationships between species richness and biomass/productivity depend on environmental factors in secondary forests of Dinghai, Zhejiang Province. Biodiversity Science, 26, 545-553. (in Chinese with English abstract) [吴初平, 韩文娟, 江波, 刘博文, 袁位高, 沈爱 华, 黄玉洁, 朱锦茹 (2018) 浙江定海次生林内物种丰富 度与生物量和生产力关系的环境依赖性. 生物多样性, 26, 545-553.]
Xiong X, Zhang HL, Wu JP, Chu GW, Zhou GY, Zhang DQ (2016) ${ }^{13} \mathrm{C}$ and ${ }^{15} \mathrm{~N}$ isotopic signatures of plant-soil continuum along a successional gradient in Dinghushan Biosphere Reserve. Chinese Journal of Plant Ecology, 40, 533-542. (in Chinese with English abstract) [熊金金, 张慧玲, 吴建平, 褚国伟, 周国逸, 张德强 (2016) 鼎湖山森林演 替序列植物-土壤碳氮同位素特征. 植物生态学报, 40, 533-542.]

Yachi S, Loreau M (2007) Does complementary resource use enhance ecosystem functioning? A model of light competition in plant communities. Ecology Letters, 10, 54-62.

Yuan Z, Ali A, Wang S, Gazol A, Freckleton R, Wang X, Lin F, Ye J, Zhou L, Hao Z, Loreau M, (2018) Abiotic and biotic determinants of coarse woody productivity in temperate mixed forests. Science of The Total Environment, 630, 422-431.

Zhang Y, Chen HYH (2015) Individual size inequality links forest diversity and Above-ground biomass. Journal of Ecology, 103, 1245-1252.

Zhang Y, Chen HYH, Reich PB (2012) Forest productivity increases with evenness, species richness and trait variation: A global meta-analysis. Journal of Ecology, 100, 742-749.

Zhang YR, Ouyang X, Chu GW, Zhang QM, Liu SZ, Zhang DQ, Li YL (2014) Spatial heterogeneity of soil organic carbon and total nitrogen in a monsoon evergreen broadleaf forest in Dinghushan, Guangdong, China. Chinese Journal of Applied Ecology, 25, 193. (in Chinese with English abstract) [张亚茹, 欧阳旭, 褚国伟, 张倩媚, 刘世忠, 张 德强, 李跃林 (2014) 鼎湖山季风常绿阔叶林土壤有机 碳和全氮的空间分布. 应用生态学报, 25, 19-23.]

Zou S, Zhou GY, Zhang QM, Xu S, Xiong X, Xia YJ, Liu SZ, Meng Z, Chu GW (2018) Long-term (1992-2015) dynamics of community composition and structure in a monsoon evergreen broad-leaved forest in Dinghushan Biosphere Reserve. Chinese Journal of Plant Ecology, 42, 442-452. (in Chinese with English abstract) [邹顺, 周国逸, 张倩媚, 徐 姗, 熊金金, 夏艳菊, 刘世忠, 孟泽, 褚国伟 (2018) 1992-2015年鼎湖山季风常绿阔叶林群落结构动态. 植物 生态学报, 42, 442-452.]

(责任编委：贺金生 责任编辑：黄祥忠) 